\title{
Towards Characterizing the Variability in the Loading Demands of an Unmanned Aerial Vehicle
}

\author{
Shankar Sankararaman* \\ SGT Inc., NASA Ames Research Center, Moffett Field, CA 94035, USA \\ Kai Goebel ${ }^{\dagger}$ \\ NASA Ames Research Center, Moffett Field, CA 94035, USA
}

\begin{abstract}
This paper presents a computational methodology to characterize and quantify the variability in the power demands during the take-off of an unmanned aerial vehicle (UAV). A lithium-ion battery-based power system is used to power the unmanned aerial vehicle, and the capabilities of the unmanned aerial vehicle are driven by the amount of charge in this battery. In order to design the power system, it is necessary to analyze the power and charge requirements of the UAV. This paper focuses on the take-off segment, and aims to quantify the amount of charge that is required for this particular segment. Sparse data is available through different flight tests and this data is used to analyze the flight profile and the charge requirement during take-off. The amount of charge required for take-off depends on several factors that are not only variable but cannot be controlled in reality, and hence, the entire flight profile and the corresponding charge requirement are variable in nature. The information available through flight tests is converted into multi-dimensional sparse data and a new method is developed in this paper for variability characterization using multi-dimensional sparse data. This analysis is useful for prognostics and health management where it is necessary to anticipate future charge requirements in order to compute the end-of-discharge of the battery, and hence, the remaining useful life of the power system.
\end{abstract}

\section{Motivation}

The use of unmanned aerial vehicles (UAVs) has considerably increased during the past ten years, owing to the development of new engineering technology and computational tools. ${ }^{1}$ The development of high power density batteries, cost-efficient airframes, and powerful microprocessors have all facilitated the use of unmanned aerial vehicles for both civilian and military operations/missions. Robustness and operational reliability are essential features of both autonomous and remotely piloted unmanned aerial vehicles. Since the performance of an unmanned aerial vehicle closely depends on its power resources, the batteries that power such unmanned aerial vehicles are of great importance. Faults in these batteries may lead to the failure of the entire power system and hence, jeopardize the entire vehicle and/or the mission. Hence, it is essential to constantly monitor the performance of the power system in order to ensure the safety of the aircraft and the success of them mission.

The field of prognostics and health monitoring (PHM) focuses on developing methods for online system health monitoring through (1) detecting and diagnosing possible system faults; (2) predicting future failures and the remaining useful life; and (3) aiding real-time decision-making activities such as fault mitigation, mission routing, path planning, etc. The application of PHM methods to unmanned aerial vehicles and power systems ${ }^{2}$ has gained increasing attention in the recent past. ${ }^{3,4}$ In particular, the use of lithium-ion batteries and health monitoring of such lithium-ion batteries have been studied in detail. ${ }^{5}$ Battery modeling techniques ${ }^{6}$ have been combined with system-level models ${ }^{7}$ for the use of prognostic tools and methods based on filtering approximations, ${ }^{8}$ analytical approximations, ${ }^{9}$ and sampling techniques. ${ }^{10}$

\footnotetext{
*Post-doctoral Research Engineer, NASA Ames Research Center, Moffett Field, CA 94035, USA.

$\dagger$ Area Lead, Discovery and Systems Health, NASA Ames Research Center, Moffett Field, CA 94035, USA
} 
A typical approach to prognostics and health management of batteries consists of two important steps: (1) an estimation step through filtering, where the state-of-charge of the battery is calculated; and (2) a prediction step, where the future discharge of the battery is computed, and the time at which end-of-discharge (EOD) occurs is calculated. In the prediction step, it is important to understand that the discharge of the battery directly depends on the future usage of the battery, which in turn depends on the future power demands of the system. In practical systems, it is almost possible to know such future power demands precisely; these power demands may depend upon several environmental factors and usage conditions many of which are not controllable. Therefore, the future power demands are uncertain and need to be treated as stochastic quantities that are variable in nature. As a result, the EOD prediction also becomes uncertain, and quantifying the uncertainty in future demands in the key to quantifying the uncertainty in the EOD prediction. The goal of this paper is to develop a computational methodology that quantifies the uncertainty in power demands, with an application to unmanned aerial vehicles.

In the context of the unmanned aerial vehicle, the future power demands are directly related to the maneuvers anticipated in the future. It is intuitive to understand that the take-off and landing segments of the UAV consume a significant amount of power and are perhaps, the most critical segments during the entire flight. Further, in general, turning consumes more power than gliding. While it may be possible to compute the exact future loading demand based on maneuvering details, such an approach requires detailed modeling of the physical system including system-level mechanics, dynamics and kinetics. This paper pursues an alternative approach, where field tests are conducted, multiple maneuvers are performed, and the power demands are measured for each of these maneuvers. To this end, a test-bed at NASA Langley Research Center $^{3}$ has been used to perform flight tests and collect information regarding the flight profile and power requirements. Once the power demands of various maneuvers can be quantified, then such information can be used for health monitoring purposes. As discussed earlier, the take-off segment is perhaps the most important and critical segment during the entire flight, and therefore, this paper focuses on quantify the power demands (and the associated uncertainty) during this segment. Other flight segments will be considered in future research.

The rest of this organized as follows. Section II discusses the data available through field tests performed at NASA Langley Research Center. Section III presents the methodology for quantifying the uncertainty in power demands, and Section IV discusses the results of uncertainty quantification. Finally, conclusions and future work are discussed in Section V.

\section{Field Data from UAV Flight Tests}

The Prognostics Center of Excellence (PCoE) at NASA Ames Research Center has been developing different types of computational methods and algorithms to facilitate diagnostics, prognostics, and online decision-making for different types of mechanical, aerospace, electrical, and civil engineering applications. In order to verify, validate, and certify these methods and algorithms, different types of test-beds have been developed. ${ }^{11-15}$ This paper utilizes data collected from the electric unmanned aerial vehicle (Fig. 1) testbed that is being used for testing different types of prognostics and decision-making algorithms at NASA Langley Research Center.

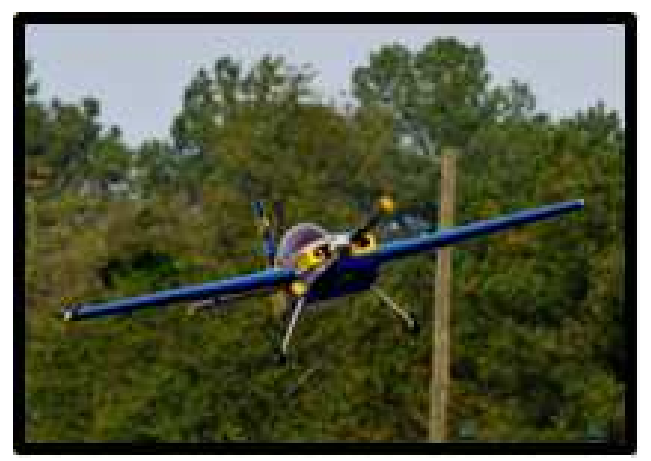

Figure 1: Unmanned Electric Vehicle: Test-Bed

Data from ten different field tests are collected; in each test, the altitude and the current are measured 
continuously as a function of time. For example, the altitude and current measurements for four of the ten field ten tests are plotted in Fig. 2 and Fig. 3 respectively.
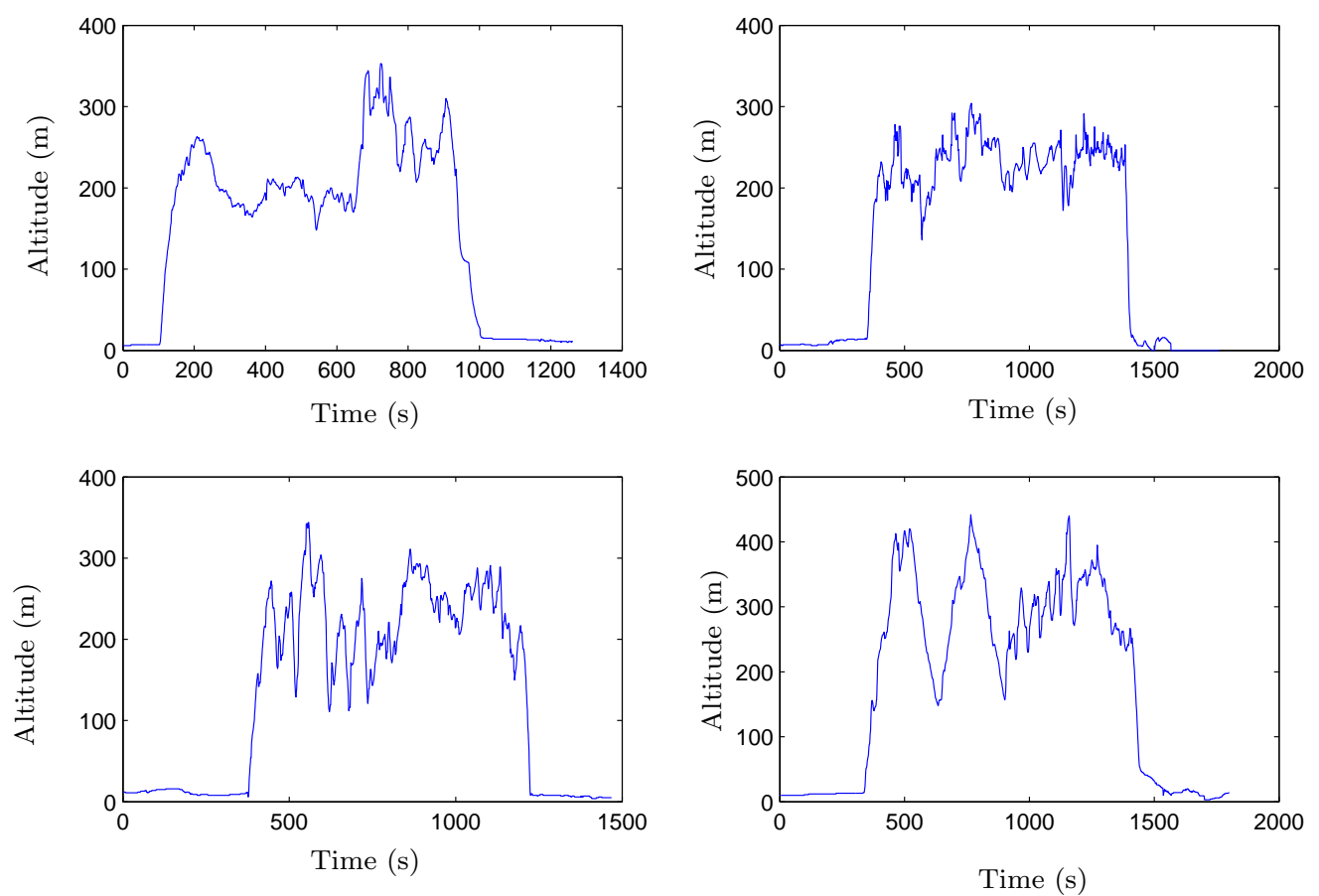

Figure 2: Time vs. Altitude

As mentioned earlier, the focus of this paper is only on the take-off segment of the unmanned aerial vehicle. The key variables are interest that completely define the flight profile and charge requirements during take-off are:

1. Start time or time at which take-off commences, assumed to be the time instant at which altitude becomes non-zero.

2. End time, defined as the time instant when a desired altitude of 150 meters is reached.

3. Peak time, the time at which the current value is maximum during take-off.

4. Duration of take-off, defined as the difference between "end time" and "start time".

5. Time to peak, defined as the difference between "peak time" and "start time".

6. Peak current, the maximum value of current during take-off

7. Total charge, calculated as the time-integral of current during the entire take-off.

For the purpose of analysis, the noise observed in the raw data is removed through moving averaging. The aforementioned quantities of interest are indicated in Table 1.

Out of the above quantities, four quantities are essential for quantifying the variability in loading demands. These four quantities include (1) Duration of take-off; (2) Time to peak; (3) Peak current; and (4) Total charge. If the variability in these quantities are quantified, then realizations of loading demands can be generated during online health monitoring, thereby directly accounting for loading variability. The next section presents a new computational approach for quantifying the variability in these quantities, and hence the variability in loading demands of the UAV. 

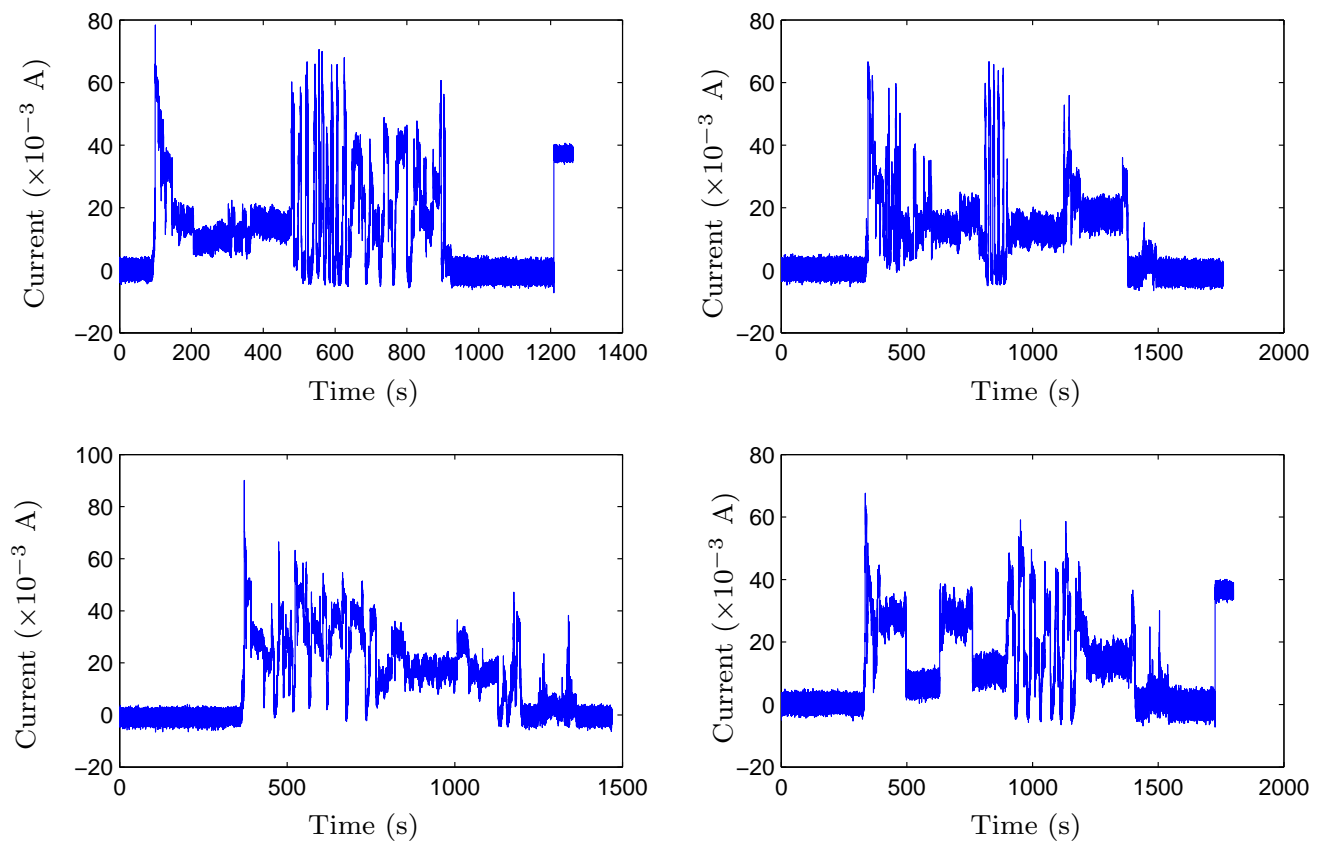

Figure 3: Time vs. Current

\section{Proposed Methodology}

The first challenge arises due to the fact that the amount of data available to characterize variability in loading conditions is sparse. This paper addresses this issue through the use of the likelihood-based approach proposed by Sankararaman and Mahadevan. ${ }^{16}$ The second challenge is that the above variables may be correlated/dependent and this cannot be directly addressed through the likelihood-based approach. For example, the correlation matrix between the four variables is given in Table 2 .

Thus, this paper develops a computational methodology to characterize variability in multi-dimensional sparsely available data. This methodology can be used to characterize the variability in the above quantities, which can then used to quantify the variability in the loading profiles of the UAV. The proposed computational methodology has three steps:

1. The first step is transform all the variables into uncorrelated space using Cholesky transformation or using a principal-component-based transformation.

Table 1: Take-off Segment: Quantities of Interest

\begin{tabular}{lccccccc}
$\begin{array}{l}\text { Quantity } \\
\text { Unit }\end{array}$ & $\begin{array}{c}\text { Start Time } \\
(\mathrm{sec})\end{array}$ & $\begin{array}{c}\text { End Time } \\
(\mathrm{sec})\end{array}$ & $\begin{array}{c}\text { Peak Time } \\
(\mathrm{sec})\end{array}$ & $\begin{array}{c}\text { Duration } \\
(\mathrm{sec})\end{array}$ & $\begin{array}{c}\text { Time to Peak } \\
(\mathrm{sec})\end{array}$ & $\begin{array}{c}\text { Peak Current } \\
(\mathrm{mA})\end{array}$ & $\begin{array}{c}\text { Total Charge } \\
(\mathrm{mA}-\mathrm{sec})\end{array}$ \\
\hline Test 1 & 90.0 & 133.0 & 99.4 & 43.0 & 9.4 & 78.4 & 1495.0 \\
Test 2 & 335.0 & 372.5 & 345.6 & 37.5 & 10.6 & 66.7 & 1257.8 \\
Test 3 & 360.0 & 403.0 & 372.2 & 43.0 & 12.2 & 90.1 & 1428.7 \\
Test 4 & 325.0 & 367.6 & 335.0 & 42.6 & 10.0 & 67.5 & 1287.5 \\
Test 5 & 552.0 & 623.0 & 561.0 & 71.0 & 9.0 & 70.8 & 1744.8 \\
Test 6 & 370.0 & 414.3 & 380.0 & 44.3 & 10.0 & 62.0 & 1179.6 \\
Test 7 & 203.0 & 241.5 & 210.7 & 38.5 & 7.7 & 74.8 & 1477.0 \\
Test 8 & 251.0 & 296.1 & 259.9 & 45.1 & 8.9 & 73.2 & 1897.8 \\
Test 9 & 210.0 & 247.3 & 215.9 & 37.3 & 5.9 & 75.3 & 1698.7 \\
Test 10 & 195.0 & 231.4 & 201.0 & 36.4 & 6.0 & 71.0 & 1652.4 \\
\hline
\end{tabular}


Table 2: Quantities of Interest: Original Correlation Matrix

\begin{tabular}{|l|cccc|}
\hline Quantity & Total Charge & Duration & Peak Current & Time to Peak \\
\hline Total Charge & 1 & 0.311 & 0.2867 & -0.5356 \\
Duration & 0.311 & 1 & -0.0671 & 0.1981 \\
Peak Current & 0.2867 & -0.0671 & 1 & 0.1945 \\
Time to Peak & -0.5356 & 0.1981 & 0.1945 & 1 \\
\hline
\end{tabular}

2. The likelihood-based methodology for uncertainty quantification is applied to each of the 4 variables in the uncorrelated space.

3. Uncorrelated samples are generated based on the results of the likelihood-based methodology, and these samples are transformed back to the original space.

The above steps are described in detail in the rest of this section, and finally, steps for verifying the results are also explained.

\section{A. Step 1: Transform into Uncorrelated Space}

In Table 1, consider the four chosen quantities (the number of variables, $n=4$ ) of interest. There are $m=10$ data samples available. Let $X$ ( $m$ by $n$ ) denote the matrix of available data.

The matrix $X$ represents correlated multi-dimensional sparse data. This matrix can be transformed into uncorrelated multi-dimensional data, using one of the two approaches mentioned below.

\section{Principal component analysis-based transform}

Consider a matrix $Z$ (size $m$ by $m$ ) whose entries are all equal to unity. Then, the uncorrelated matrix $U$ (size $m$ by $n$ ) can be obtained using the following two steps, based on Principal Component Analysis (PCA):

$$
\begin{gathered}
D=X-\frac{1}{m} Z_{m, m} X_{m, n} \\
U=D\left(D^{T} D\right)^{-\frac{1}{2}}
\end{gathered}
$$

\section{Cholesky-based transform}

Consider the covariance matrix of $X$, denoted by $\Sigma_{n, n}$. This covariance can be written in terms of a lower triangular matrix $L$ as:

$$
\Sigma=L L^{T}
$$

Using the matrix $L$, the uncorrelated matrix $U$ can be computed as:

$$
U=\left(L^{-1} X^{T}\right)^{T}
$$

\section{B. Step 2: Likelihood-based Method for Uncertainty Quantification}

The uncorrelated multi-dimensional matrix can then be used for uncertainty quantification, where each variable in $U$ can be treated separately; the $j^{\text {th }}$ column in $U$ corresponds to the $j^{\text {th }}$ variable $(j=1$ to $n$, and $n=4$ in this application).

Choose a probability distribution type, whose probability density function (PDF) is indicated by $f_{X}(x \mid \boldsymbol{P})$ where $\boldsymbol{P}$ denotes the distribution parameters. A probability distribution type is chosen for the $j^{\text {th }}$ variable and the parameters are estimated using the principle of likelihood, ${ }^{16}$ as:

$$
L(\boldsymbol{P}) \propto \prod_{i=1}^{i=m} f_{X}\left(x=U_{i, j} \mid \boldsymbol{P}\right)
$$

assuming that the $m$ field tests are independent of each other. 
The maximum likelihood estimate (MLE) of the parameters can be estimated by selecting the value of $\boldsymbol{P}$ that maximizes Eq. 5. While the MLE method presents one approach for parameter treatment, the entire probability distribution of the parameters can be alternatively estimated using Bayesian inference (choose uniform prior density, as $\left.f_{\boldsymbol{P}}^{\prime}(\boldsymbol{p})=h\right)$, as:

$$
f_{\boldsymbol{P}}(\boldsymbol{p})=\frac{h L(\boldsymbol{p})}{\int h L(\boldsymbol{p}) d \boldsymbol{p}}=\frac{L(\boldsymbol{p})}{\int L(\boldsymbol{p}) d \boldsymbol{p}}
$$

Note that $\boldsymbol{p}$ is a realization of the random variable $\boldsymbol{P}$. The uniform prior density function can be defined over the entire admissible range of the parameters $\boldsymbol{P}$. For example, the mean of a normal distribution can vary in $(-\infty, \infty)$ while the standard deviation can vary in $(0, \infty)$ because the standard deviation is always greater than zero. Both these prior distributions are improper prior distributions because they do not have finite bounds.

A single unconditional PDF of $X$, which includes both the variability in $X$ and the uncertainty in the distribution parameters $\boldsymbol{P}$, can be computed as:

$$
f_{X}(x)=\int f_{X}(x \mid \boldsymbol{p}) f_{\boldsymbol{P}}(\boldsymbol{p}) d \boldsymbol{p}
$$

Note that the RHS of Eq. 7 is not conditioned on $\boldsymbol{P}$ anymore. Some researchers refer to this PDF $f_{X}(x)$ as the predictive $\mathrm{PDF}^{17}$ of $X$. The predictive distribution approach is the second choice for parameter treatment.

During implementation, it is necessary to choose (1) the distribution type for each variable (choice of $f_{X}(x \mid \boldsymbol{P})$ ); and (2) the choice of parameter treatment (MLE vs predictive). The above procedure is repeated for each of the $n$ variables, and the probability density function for each of these variables is calculated. Based on these probability distributions, samples for each variable can be generated independently of the probability density functions of the other variables, since this computation is performed in the uncorrelated space. Several thousands of samples can be generated, and the result matrix is denoted as $V$ (size $m$ by $N$, where $N>>n$ ).

\section{Step 3: Transform into Original Space}

Finally, the matrix $V$ is transformed back into the original space using the inverse Cholesky-based transform (i.e., by inverting Eq. 3 and Eq. 2) or the inverse principal component analysis-based transform (i.e., by inverting Eq. 1 and Eq. 2). The resultant matrix in the original space is denoted by $Y$.

For the purpose of generating an arbitrary realization of the power demand trajectory, an arbitrary realization of samples is generated in the uncorrelated space ( $V$-space), and transformed into the original space ( $Y$-space); then the resultant information can be used to construct the power demand trajectory during take-off. Several such realizations can be easily generated to represent the uncertainty in these trajectories and hence used to calculate the uncertainty in the end-of-discharge prediction and the remaining useful life prediction.

\section{Verification}

An important task is to verify the result of the above described uncertainty quantification methodology. A two step verification is performed in this paper.

First, the marginal distribution of each variable after uncertainty quantification is compared with the marginal distribution before uncertainty quantification. The former is computed based on the $N$ samples generated in Section C. The latter is computed by applying the likelihood-based method to the original values in $X$; in order to facilitate fair comparison, the same distribution type and the same parameter treatment is chosen for both former and latter. Second, the correlation matrix of $Y$ is compared against the original correlation matrix of $X$ (in Table 2). It is important that both the marginal distributions and the correlation matrix before and after transformation compare well against each other. Thereby, the variability in each of the quantities of interest can be accurately quantified, along with the correlation/dependence information amongst the quantities of interest. The numerical results of uncertainty quantification and verification results are indicated in Section IV. 


\section{Numerical Results}

The results of uncertainty quantification depend on:

1. The choice of the transform from correlated space to uncorrelated space;

2. Choice of distribution type for each variable; and

3. Choice of parameter treatment (MLE vs. predictive)

As discussed earlier in this paper, there are two choices for the first and third categories above. However, the choice of distribution type is subjective in nature. While an extensive set of combinations were tested, set of results for uncertainty quantification and verification are presented for the following choices for each of the above categories:

1. Choice I - Normal distribution, MLE, PCA-based transform

2. Choice II - Truncated normal distribution, Predictive, PCA-based transform

3. Choice III - Johnson (Bounded) distribution, Predictive, Cholesky-based transform

These results are presented in terms of (1) marginal probability density functions before and after transformation; (2) correlation matrix after transformation that can be compared against Table 2.

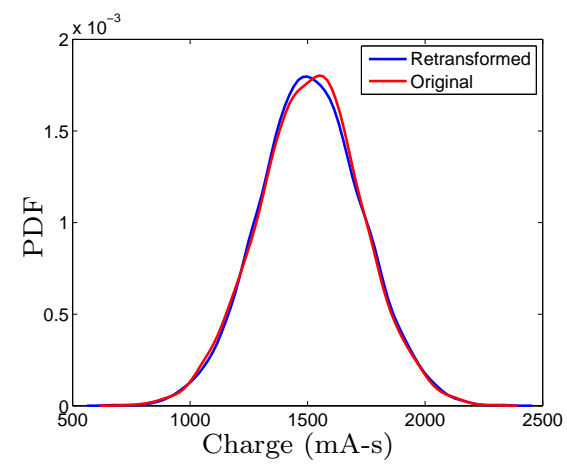

(a) Total Charge

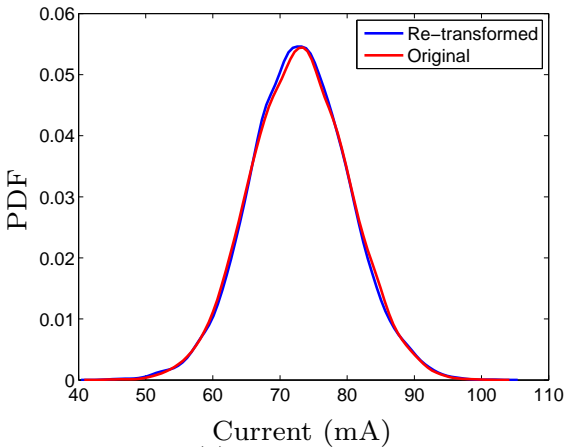

(c) Peak Current

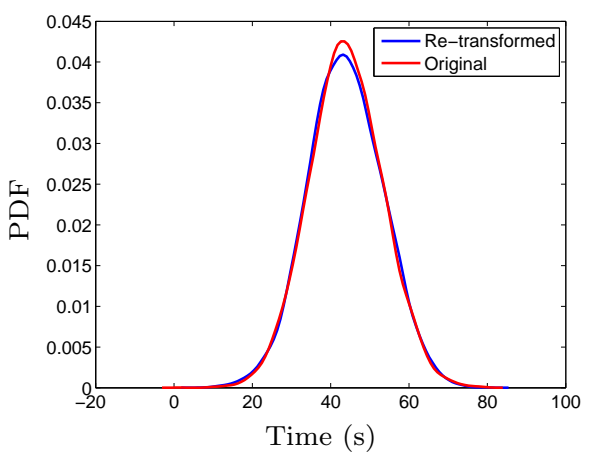

(b) Take-off Duration

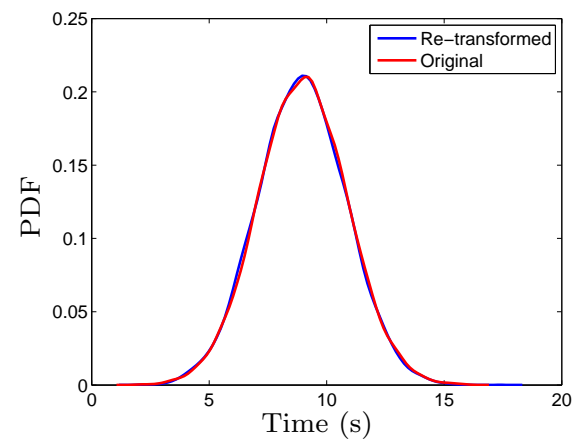

(d) Time to Peak

Figure 4: PDFs of Quantities of Interest: Choice I

In general, it was noted that the results from Cholesky-based transformation and PCA-based transformation were almost identical with one another. Further, it can be seen from the above figures and tables that verification procedure was reasonably successful as the marginal distributions before and after transformation match reasonable well with another, and further, the correlation structure before and after transformation also matches well. The reason for differences can be attributed to the fact that it is almost practically impossible to draw random correlated samples from joint probability distributions. If the joint distribution 
Table 3: Choice I: Correlation Matrix after Transformation

\begin{tabular}{|l|cccc|}
\hline Quantity & Total Charge & Duration & Peak Current & Time to Peak \\
\hline Total Charge & 1 & 0.3179 & 0.2816 & -0.5279 \\
Duration & 0.3179 & 1 & -0.0593 & 0.1981 \\
Peak Current & 0.2816 & -0.0593 & 1 & 0.2045 \\
Time to Peak & -0.5279 & 0.1981 & 0.2045 & 1 \\
\hline
\end{tabular}

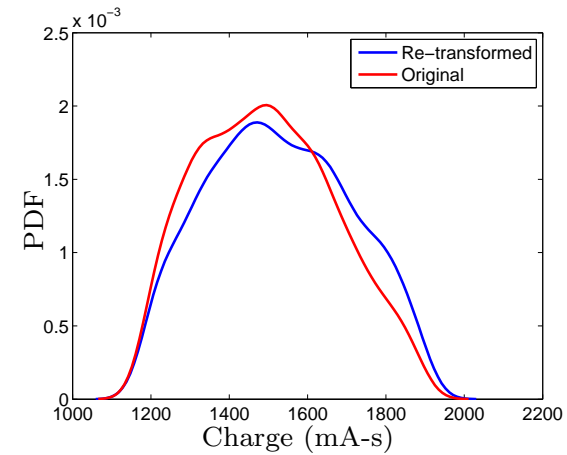

(a) Total Charge

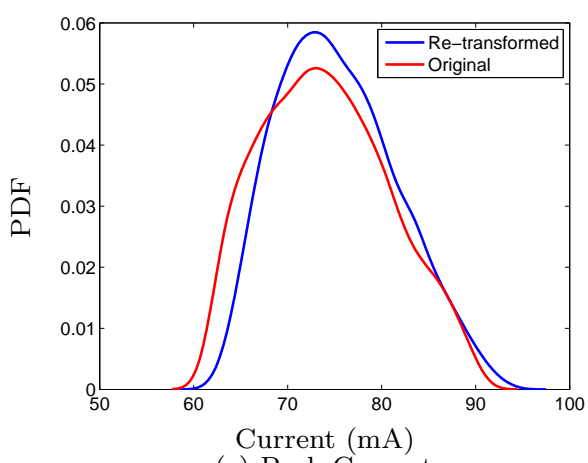

(c) Peak Current

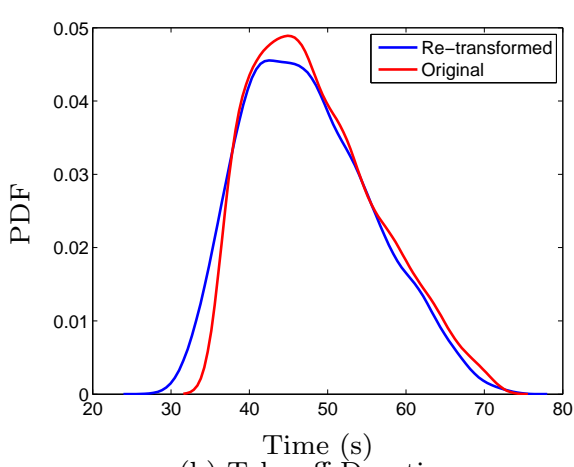

(b) Take-off Duration

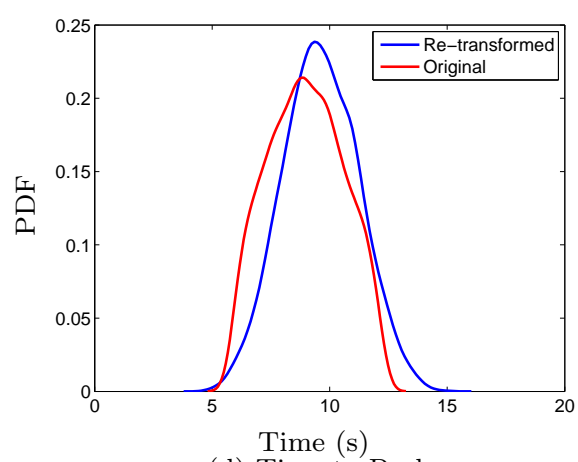

(d) Time to Peak

Figure 5: PDFs of Quantities of Interest: Choice II

is normal, then it is possible to draw samples that exactly match with both the individual marginal distributions and the correlation structure (as seen from the results of Choice-I); however, for arbitrary joint distributions, it is possible to either match the individual marginal distributions or the correlation structure but not both (as seen from the results of Choice-II and Choice-III).

While this paper addressed variability in the take-off loading segment, this research needs to be continued in order to quantify the uncertainty in the entire loading trajectory. Then, it will be possible to estimate the end-of-discharge as a continuous function of time. This may be in considered in future work.

\section{Conclusion}

This paper presented a computational approach to quantify the variability in the power demands of an unmanned aerial vehicle. Field data was collected using an unmanned electric vehicle as a test-bed at NASA Langley Research Center. Ten different flight tests were conducted and in each test, the altitude and the current was measured continuously as a function of time. In order to generate a realization of the future loading demand, four quantities of interest - duration of take-off, total charge required, maximum current, time to peak - were identified for uncertainty quantification. In order to account for possible correlation/dependence between the variables, they were first transformed to an uncorrelated space; a Cholesky-based transformation 
Table 4: Choice II: Correlation Matrix after Transformation

\begin{tabular}{|l|cccc|}
\hline Quantity & Total Charge & Duration & Peak Current & Time to Peak \\
\hline Total Charge & 1 & 0.2835 & 0.2738 & -0.523 \\
Duration & 0.2835 & 1 & -0.0644 & 0.2321 \\
Peak Current & 0.2738 & -0.0644 & 1 & 0.2199 \\
Time to Peak & -0.523 & 0.2321 & 0.2199 & 1 \\
\hline
\end{tabular}

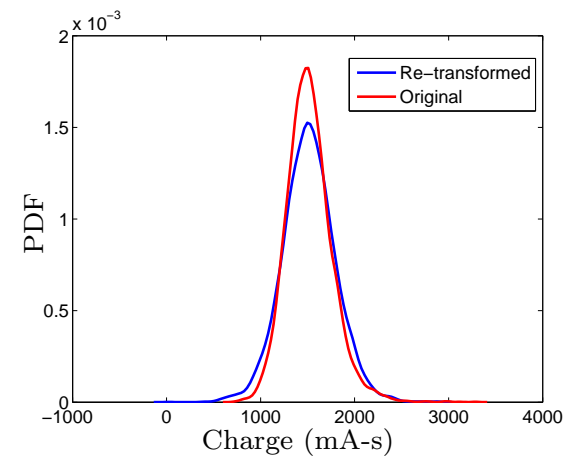

(a) Total Charge

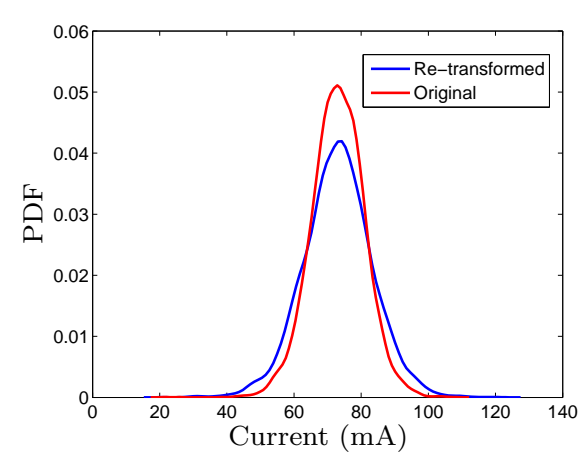

(c) Peak Current

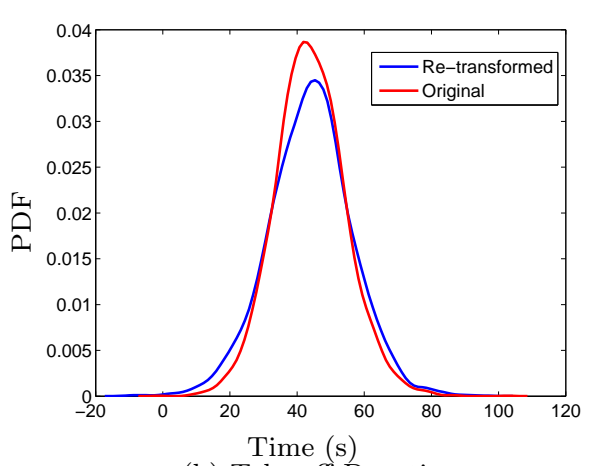

(b) Take-off Duration

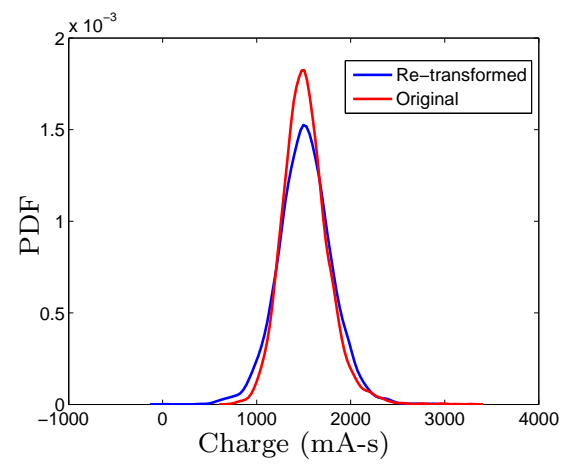

(d) Time to Peak

Figure 6: PDFs of Quantities of Interest: Choice III

scheme and a principal component analysis-based transformation approach were discussed for this purpose. In the uncorrelated space, a likelihood-based methodology was used to quantify the uncertainty in the four quantities of interest. After such uncertainty quantification, the variables were transformed back into the original space. The results of the uncertainty quantification methodology were verified by comparing (1) the original probability distribution of each variable against the probability distribution obtained after transformation; and (2) the original correlation matrix against the correlation matrix obtained after variability characterization.

There are several possible directions for future work. This paper focused on quantifying the variability only during the take-off segment of the flight. Further research needs to account for variability in loading demands during other maneuvers such as ascent, descent, gliding, turning, landing, etc. This will facilitate the generation of realizations of the entire loading trajectory, which is helpful for end-of-discharge prediction and remaining useful life prediction during system health management; this information will also be useful for online decision-making. 
Table 5: Choice III: Correlation Matrix after Transformation

\begin{tabular}{|l|cccc|}
\hline Quantity & Total Charge & Duration & Peak Current & Time to Peak \\
\hline Total Charge & 1 & 0.2972 & 0.2405 & -0.5341 \\
Duration & 0.2972 & 1 & -0.0932 & 0.1948 \\
Peak Current & 0.2405 & -0.0932 & 1 & 0.252 \\
Time to Peak & -0.5341 & 0.1948 & 0.252 & 1 \\
\hline
\end{tabular}

\section{Acknowledgment}

The work reported herein was in part funded by the NASA System-wide Satefy Assurance Technologies (SSAT) project under the Aviation Safety (AvSafe) Program of the Aeronautics Research Mission Directorate (ARMD). The authors would also like to thank Dr. Brian Bole at NASA Ames Research Center for collecting flight test field data from NASA Langley Research Center.

\section{References}

${ }^{1}$ Chao, H., Cao, Y., and Chen, Y., "Autopilots for small unmanned aerial vehicles: a survey," International Journal of Control, Automation and Systems, Vol. 8, No. 1, 2010, pp. 36-44.

${ }^{2}$ Zhang, J. and Lee, J., "A review on prognostics and health monitoring of Li-ion battery," Journal of Power Sources, Vol. 196, No. 15, 2011, pp. 6007-6014.

${ }^{3}$ Balaban, E. and Alonso, J. J., "An Approach to Prognostic Decision Making in the Aerospace Domain," Annual Conference of the Prognostics and Health Management Society, 2012.

${ }^{4}$ Balaban, E. and Alonso, J. J., "A Modeling Framework for Prognostic Decision Making and its Application to UAV Mission Planning," Annual Conference of the Prognostics and Health Management Society, 2013.

${ }^{5}$ Hatzell, K. B., Sharma, A., and Fathy, H. K., "A survey of long-term health modeling, estimation, and control of Lithium-ion batteries: Challenges and opportunities," American Control Conference (ACC), 2012, IEEE, 2012 , pp. 584-591.

${ }^{6}$ Gould, C. R., Bingham, C. M., Stone, D. A., and Bentley, P., "New battery model and state-of-health determination through subspace parameter estimation and state-observer techniques," Vehicular Technology, IEEE Transactions on, Vol. 58, No. 8, 2009, pp. 3905-3916.

${ }^{7}$ Daigle, M. and Kulkarni, C. S., "A battery health monitoring framework for planetary rovers," Aerospace Conference, 2014 IEEE, IEEE, 2014, pp. 1-9.

${ }^{8}$ Saha, B., Goebel, K., Poll, S., and Christophersen, J., "Prognostics methods for battery health monitoring using a Bayesian framework," Instrumentation and Measurement, IEEE Transactions on, Vol. 58, No. 2, 2009, pp. $291-296$.

${ }^{9}$ Sankararaman, S., Daigle, M., and Goebel, K., "Uncertainty Quantification in Remaining Useful Life Prediction Using First-Order Reliability Methods," Reliability, IEEE Transactions on, Vol. 63, No. 2, June 2014, pp. 603-619.

${ }^{10}$ Sankararaman, S., "Significance, interpretation, and quantification of uncertainty in prognostics and remaining useful life prediction," Mechanical Systems and Signal Processing, Vol. 5253, No. 0, 2015, pp. $228-247$.

${ }^{11}$ Poll, S., Patterson-Hine, A., Camisa, J., Garcia, D., Hall, D., Lee, C., Mengshoel, O. J., Neukom, C., Nishikawa, D., Ossenfort, J., et al., "Advanced diagnostics and prognostics testbed," Proceedings of the 18th International Workshop on Principles of Diagnosis (DX-07), 2007, pp. 178-185.

${ }^{12}$ Balaban, E., Narasimhan, S., Daigle, M., Roychoudhury, I., Sweet, A., Bond, C., and Gorospe, G., "Development of a mobile robot test platform and methods for validation of prognostics-enabled decision making algorithms," International Journal of Prognostics and Health Management, Vol. 4, No. 1, 2013.

${ }^{13}$ Kulkarni, C. S., Daigle, M., Gorospe, G., and Goebel, K., "Validation of Model-Based Prognostics for Pneumatic Valves in a Demonstration Testbed," 2014.

${ }^{14}$ Kulkarni, C. S., Gorospe, G., Daigle, M., and Goebel, K., "A testbed for implementing prognostic methodologies on cryogenic propellant loading systems," AUTOTESTCON, 2014 IEEE, IEEE, 2014, pp. 280-289.

${ }^{15}$ Bole, B., Teubert, C., Chi, Q., Edward, H., Vazquez, S., Goebel, K., and Vachtsevanos, G., "SIL/HIL replication of electric aircraft powertrain dynamics and inner-loop control for $\mathrm{V} \& \mathrm{~V}$ of system health management routines," Annual conference of the prognostics and health management society, 2013.

${ }^{16}$ Sankararaman, S. and Mahadevan, S., "Likelihood-based representation of epistemic uncertainty due to sparse point data and/or interval data," Reliability Engineering 85 System Safety, Vol. 96, No. 7, 2011, pp. 814-824.

${ }^{17}$ Der Kiureghian, A., "Measures of structural safety under imperfect states of knowledge," Journal of Structural Engineering, Vol. 115, No. 5, 1989, pp. 1119-1140. 